\title{
Wigner term generated by isovector p-n pairing
}

\author{
Ian Bentley ${ }^{1,2, a}$ and Stefan Frauendorf ${ }^{1, b}$ \\ ${ }^{1}$ Department of Physics, University of Notre Dame, Notre Dame, IN 46556 \\ ${ }^{2}$ Department of Chemistry and Physics, Saint Mary's College, Notre Dame, IN 46556
}

\begin{abstract}
The linear term proportional to $|N-Z|$ in the nuclear symmetry energy (Wigner energy) is obtained in a model that uses isovector pairing on single particle levels from a deformed potential combined with a $\vec{T}^{2}$ interaction. The pairing correlations are calculated by numerical diagonalization of the pairing Hamiltonian acting on the six or seven levels nearest the $N=Z$ Fermi surface. The experimental binding energies of nuclei with $N \approx Z$ are well reproduced. The Wigner energy emerges as a consequence of restoring isospin symmetry.
\end{abstract}

\section{Introduction}

The nuclear ground state energy, $E(N, Z)$, as a function of the proton number $(Z)$ and neutron number $(N)$ or atomic mass number $(A=N+Z)$ is very well described by the celebrated empirical mass formula (see e.g. [1]):

$$
E(N, Z)=E_{V}+E_{S}+E_{C}+E_{P}+E_{A}+E_{W}+E_{S H E L L} .
$$

The first four terms account for the volume, surface, coulomb and pairing. The (a-)symmetry energy, $E_{A}=$ $a_{A}(N-Z)^{2} / A$, consists of two approximately equal contributions. The "kinetic"part accounts for the Pauli principle, which requires the nucleons to occupy higher single particle levels with increasing asymmetry $|N-Z|$. The "interaction"part originates from the difference between the proton-proton, neutron-neutron and proton-neutron interactions. The pairing energy $\left(E_{P}\right)$ describes the energy gain by forming pairs of protons or neutrons. The term, $E_{W}=a_{W}|N-Z| / A$, is called the Wigner energy, because Wigner [2] gave a first interpretation in terms of his super multiplet theory. However, its physical origin has been the subject of a long debate, which has been recently reviewed by [3]. The shell energy $\left(E_{S H E L L}\right)$ is a manifestation of the level bunching around the Fermi level.

Modern mean field approaches reproduce the ground state energies very well, except the Wigner energy, which has to be added as an ad-hoc phenomenological term (see e.g. [4]). This means that the physics behind the Wigner energy is not taken into account by present mean field theories.

As the ground state isospin $(T)$ of most nuclei is equal to their isospin projection $\left(T_{z}=\frac{N-Z}{2}\right)$, the sum of the symmetry and Wigner energies is approximately proportional to $T(T+1)$. The $T$ - dependence is suggestive, because the isospin operators obey the same $\mathrm{SU}_{2}$ algebra as the angular momentum operators. Spontaneous breaking of the

\footnotetext{
a e-mail: ibentley@saintmarys.edu

be-mail: sfrauend@nd.edu
}

rotational symmetry by the deformed mean field leads to the appearance of rotational bands. The energies of the rotational levels are proportional to $I(I+1)$, with $I$ being the angular momentum. The analogy between nuclear spin and isospin led Frauendorf and Sheikh [5, 6] to suggest that the $T(T+1)$ dependence of the ground state energy is a manifestation of an isorotational band.

The band appears because the isovector pair field, which is a vector, spontaneously breaks rotational symmetry in isospace. In the limit of strong symmetry breaking, simply the isorotational energy $T(T+1) / 2 \Theta$ is added to the intrinsic energy of the symmetry breaking mean field, the orientation of which can be taken such that the protonneutron pair field is zero [5, 6]. Afanasjev et al. [7-9] successfully used this simple limit to interpret the excitation spectra of nuclei with $N \approx Z$.

In a series of papers, Jänecke and coworkers [10] (and earlier work cited therein) demonstrated that the global $N-, Z-$ dependence of the binding energies, including the Wigner term and the inversion of the $T=0$ and $T=1$ states in odd-odd $N=Z$ nuclei with $A>40$, can be well understood in terms of the competition between the familiar pair gap $\Delta$ and a symmetry energy term of the form $T(T+1)$.

In the present work we put this qualitative interpretation on a microscopic foundation, which will allow us to make predictions how the Wigner energy depends on deformation (inclusive fission), on angular momentum and on temperature.

\section{Isolating the Wigner $X$ from experiment}

As a starting point we assume that the isospin mixing caused by the Coulomb interaction can be neglected. Ref. [11] estimates the admixture of components with $T>T_{z}$ to the ground state to be of the order of $0.9 \%$ for $A \sim 70$. With this assumption, the Coulomb energy can be separated from the energy caused by the strong interaction. 
Following [10] we subtract the Coulomb energy from the experimental energies and compare the resulting energies with our model.

The expression for the Coulomb energy given in Ref. [12] was adopted. A fit to the experimental binding energies of 69 pairs of mirror nuclei from the 2012 Atomic Mass Evaluation (AME2012) [13] was used to determine the two parameters $r_{0}=1.224 \mathrm{fm}$ and $d=0.397 \mathrm{fm}$. The resulting expression for the Coulomb energy:

$$
E_{C}=0.706 \frac{Z^{2}}{A^{1 / 3}}-0.305 \frac{Z^{2}}{A}-0.539 \frac{Z^{4 / 3}}{A^{1 / 3}}[\mathrm{MeV}]
$$

was used to determine experimental strong interaction energies defined by $E_{S}(N, Z)=E_{E x p}(N, Z)-E_{C}(N, Z)$, where $E_{E x p}(N, Z)$ were taken from the AME2012.

In accordance with the concept of isorotational bands, we write the energy of an isobaric chain, with constant $A$, in the form:

$$
E(N, Z)=E_{\text {int }}+\frac{T(T+X)}{2 \Theta}, T=\left|T_{z}\right|,
$$

where, $E_{\text {int }}$ is the energy of the intrinsic $(N=Z)$ configuration. The term $1 / 2 \Theta$ is a combination of the coefficient $a_{S}$ of the the symmetry energy and a contribution from the shell energy $E_{S H E L L}(Z, N)$, which depends on $T_{z}$. Likewise, $X / \Theta$ is a combination related to the coefficient $a_{W}$ of the Wigner energy, which also contains a contribution from $E_{S H E L L}$.

\section{Model calculations}

A monopole isovector Hamiltonian is used to describe the pair correlated ground state,

$$
\begin{gathered}
H_{V}=\sum_{k} \epsilon_{k} \hat{N}_{k}-G \sum_{k k^{\prime}, \tau} \hat{P}_{k, \tau}^{+} \hat{P}_{k^{\prime}, \tau}+C \vec{T} \cdot \vec{T}, \\
\hat{N}_{k}=\hat{p}_{k}^{+} \hat{p}_{k}+\hat{p}_{\bar{k}}^{+} \hat{p}_{\bar{k}}+\hat{n}_{k}^{+} \hat{n}_{k}+\hat{n}_{\bar{k}}^{+} \hat{n}_{\bar{k}}, \\
\hat{P}_{k,-1}^{+}=\hat{p}_{k}^{+} \hat{p}_{\bar{k}}^{+}, \hat{P}_{k, 1}^{+}=\hat{n}_{k}^{+} \hat{n}_{\bar{k}}^{+}, \\
\text {and } \hat{P}_{k, 0}^{+}=\frac{1}{\sqrt{2}}\left(\hat{n}_{k}^{+} \hat{p}_{\bar{k}}^{+}+\hat{p}_{k}^{+} \hat{n}_{\bar{k}}^{+}\right),
\end{gathered}
$$

where $\hat{p}_{k}^{+}$and $\hat{n}_{k}^{+}$create a proton and a neutron, respectively, on the level $k$. The time reversed state of $k$ is denoted by $\bar{k}$. This Hamiltonian is invariant under rotations in isospace, i.e. it conserves isospin. As suggested by Neergård [3], the term $C \vec{T} \cdot \vec{T}$ is a simple way to take into account the isospin dependence of the single particle levels. The relation between the isospin dependence of the nuclear potential and the "interaction"part of the symmetry energy has been discussed by Bohr and Mottelson [14]. It needs to be added, because we carry out the diagonalization of the pairing Hamiltonian for a fixed set of single particle levels along an isobaric chain. This means that only the "kinetic"part of the symmetry energy is taken into account.

The single particle energies used in the pairing calculations are calculated by means of the Micro-Macro method using a Nilsson Hamiltonian as described in Ref. [15]. For each nucleus the equilibrium deformation has been calculated. Ref. [16] discusses this procedure of determining the equilibrium deformation called AutoTAC in more detail. The single particle energies used in the diagonalization of the pairing Hamiltonian are taken as the average of the proton and neutron energies calculated by the Nilsson model at equilibrium deformation.

The premise of our model is that isospin is conserved, i.e. the relative energies of the proton levels and the relative energies of the neutron levels must be the same. For this reason, we chose to take the average. An overall shift of the proton levels by a constant energy results only in a constant shift of the average single particle energy, which does not matter, because we only consider energy differences. The model contains two parameters, strength of the isovector pair interaction $G$, and the parameter $C$ of the "symmetry"interaction, which have been determined by simultaneously by fitting the even-even odd-odd mass differences and the energy difference between the $T=0$ and $T=1$ states in the odd-odd nuclei.

The coupling constant $G(A)$ was adjusted to reproduce the experimental values of the even-even odd-odd mass differences of the $T=0$ states in $N=Z$ nuclei using the 3-point formula:

$$
2 \Delta(N, Z)=\frac{B_{E}(\mathrm{~N}-1, \mathrm{Z}-1)-2 B_{E}(\mathrm{~N}, \mathrm{Z})-B_{E}(\mathrm{~N}+1, \mathrm{Z}+1)}{2},
$$

which was evaluated using the binding energies of the $T=0$ states in the $N=Z$ nuclides. The following fit was adopted: $G=13.9 A^{-3 / 4}[\mathrm{MeV}]$. Figure 1 compares the experimental values with the ones obtained from calculated energies using $G(A)$ and Nilsson levels corresponding to AutoTAC equilibrium deformation. In calculating the odd-odd nuclei, the fourth (middle) level was blocked, because the $T=0$ states have two quasi-particle character with respect to isovector pair correlations. Overall, there is good agreement. The deviations are likely a result of inaccuracies of the Nilsson levels. The deformations resulting from the AutoTAC calculation are often substantially smaller than those determined experimentally using $B(E 2)$ values.

The energy difference $E(T=1)-E(T=0)$ in the oddodd $N=Z$ was then used to fix the parameter $C$ Without the symmetry interaction term, the fully correlated $T=1$ state lies at least $2 \Delta$ below the blocked $T=0$ state. However, the inclusion of the symmetry interaction term ( $2 C$ is added only to the $T=1$ state) results in comparable energies for the two states. Depending on $A$ and the two states can switch order, which is seen experimentally. Requiring the smooth $1 / A$-dependence of the symmetry energy, the fit of the calculated differences $E(T=1)-E(T=0)$ to the experimental ones gave: $C=58.9 A^{-1}[\mathrm{MeV}]$.

Figure 1 shows that calculations well reproduce energy difference between the lowest $T=1$ and $T=0$ states, which measures to relative strength of the isovector pair correlation and the isorotational energies. There are large fluctuations in the theoretical calculations caused by the uneven level spacings, which are roughly reproduced. The deviations are of the same order as the ones of $2 \Delta$ and have the same origin. 

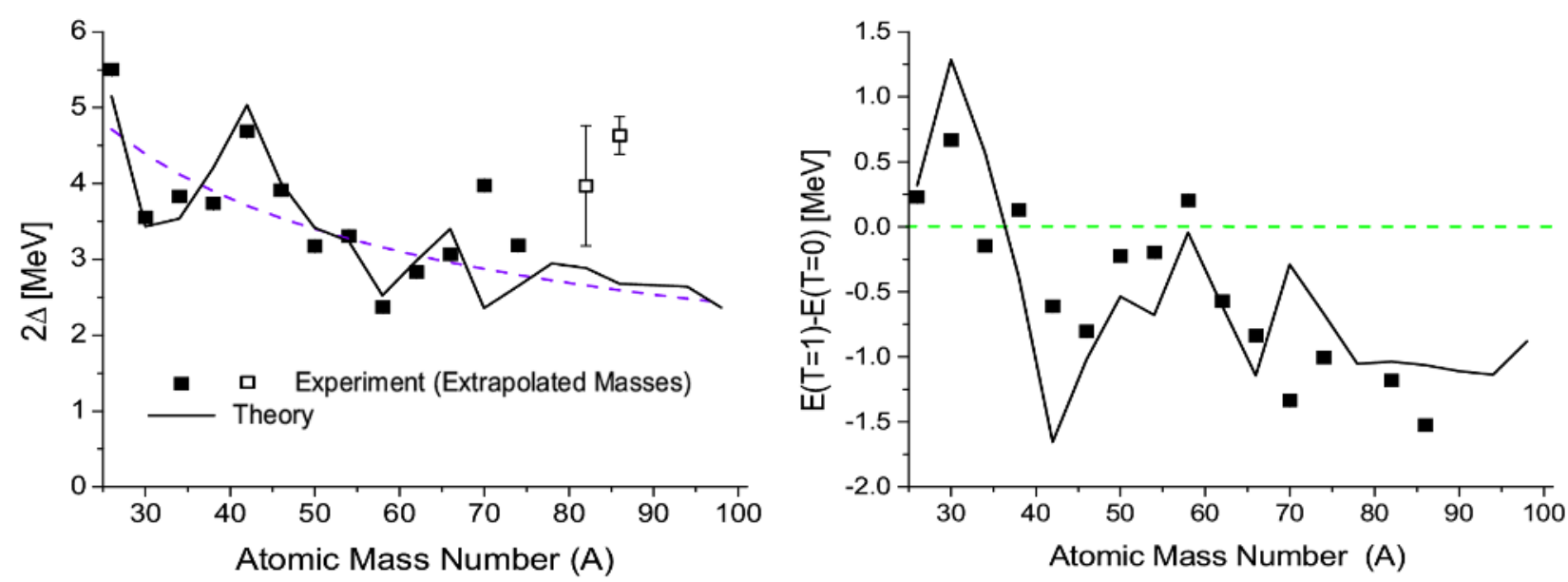

Figure 1. (Left) The even-even odd-odd mass difference $2 \Delta$ obtained from (7) using modified energies from [13] with the Coulomb energy removed. The solid line shows the calculations. The purple dashed line is the global fit of $2 \Delta=24 A^{-1 / 2}[\mathrm{MeV}]$. (Right) Energy difference of the first $T=1$ from the first $T=0$ states in odd-odd $\mathrm{N}=\mathrm{Z}$ nuclei. The solid line shows the calculations. Experimental data from NNDC [18]. The green dashed line indicates where inversion occurs.

The coefficient of the symmetry energy in the liquid drop model $4 a_{A}=1 / 2 \theta_{L D}=100 \mathrm{MeV} / \mathrm{A}$. The value of $C=$ $58.9 \mathrm{MeV} / \mathrm{A}$ is consistent with the general estimates [14] that the interaction part of the symmetry energy amounts to about $50 \%$ of its total value.

\section{The isorotational moment of inertia and Wigner $X$}

Combining the energy differences as:

$$
\frac{1}{\theta}=\frac{E_{S}\left(T_{z}=0,1\right)-2 E_{S}\left(T_{z}=2,3\right)+E_{S}\left(T_{z}=4,5\right)}{4},
$$

the local slope for both the even and odd chains of $T_{z}$ was calculated. It has the meaning of the inverse moment of inertia of the isorotational sequence. Figure 2 displays a comparison between experiment and the theoretical calculation of the slope.

The calculated values of $1 / \theta$ are systematically somewhat larger than the experimental ones. We think that this reflects a fringe effect of our small single particle space. The studies of Refs. [10] and [17] demonstrated that the experimental values of $1 / \theta$ and $2 \Delta$ are consistent with $E(T=1)-E(T=0)=2 \Delta-1 / \theta$ on average. We expect that including more single particle levels into the beyondmean field description of the pair correlations will resolve the modest inconsistency.

Figure 2 demonstrates that the calculations reproduce well the observed values of $X$, both the average, which is somewhat larger than 1 , and the pronounced fluctuations. The amplitude of the fluctuations increases with the degree of bunching of the levels. Strong bunching occurs near a doubly magic numbers 56 and 92 due to the degeneracy of the spherical levels, which causes the large fluctuations. In the region $A \approx 56$, the $X$ values overestimate their amplitude. The calculated AutoTAC deformations in this region are zero. However, one expects that the degeneracy of the spherical levels will be lifted by coupling to shape vibrations. This which will damp the fluctuations.

There is a tendency that the calculations underestimate $X$ for $74 \leq A \leq 92$. On the one hand, the experimental uncertainties are large in this mass region, and several binding energies are extrapolated. On the other, the calculated AutoTAC deformations are moderate and fairly constant, which results in $X$ values close to one. The experimental yrast energies of these nuclei change from more rotational to more vibrational behavior as $T_{z}$ increases. This points to a more rapid change of deformation than calculated by AutoTAC. We calculated $X$ assuming a substantial decrease of the deformation along an isobaric chain and found that $X$ increased enough to explain the discrepancy.

\section{Conclusions}

We have studied a model based on single particle levels in a deformed potential, isospin conserving isovector monopole pairing, and a schematic "symmetry interaction" proportional to $\vec{T}^{2}$. The pairing correlations are treated exactly by numerical diagonalization in a space of seven single particle levels, which ensured that isospin is conserved. The model does not introduce new parameters as compared to standard mean field approaches. The two model parameters are the pairing strength, which is fixed by the even-even to odd-odd mass difference, and the strength of the symmetry interaction, which is determined by the energy difference between the lowest $T=0$ and $T=1$ states in odd-odd $N=Z$ nuclei. Assuming a smooth $A$ - dependence for both parameters the model reproduces the experiment within few hundred $\mathrm{keV}$.

Merging the symmetry term and the Wigner term (linear in $|N-Z|)$ in the nuclear binding to one expression of the form $T(T+X) / 2 \theta$, the values of $X$ are found to scatter around 1 . The Wigner term appears as a result of breaking 

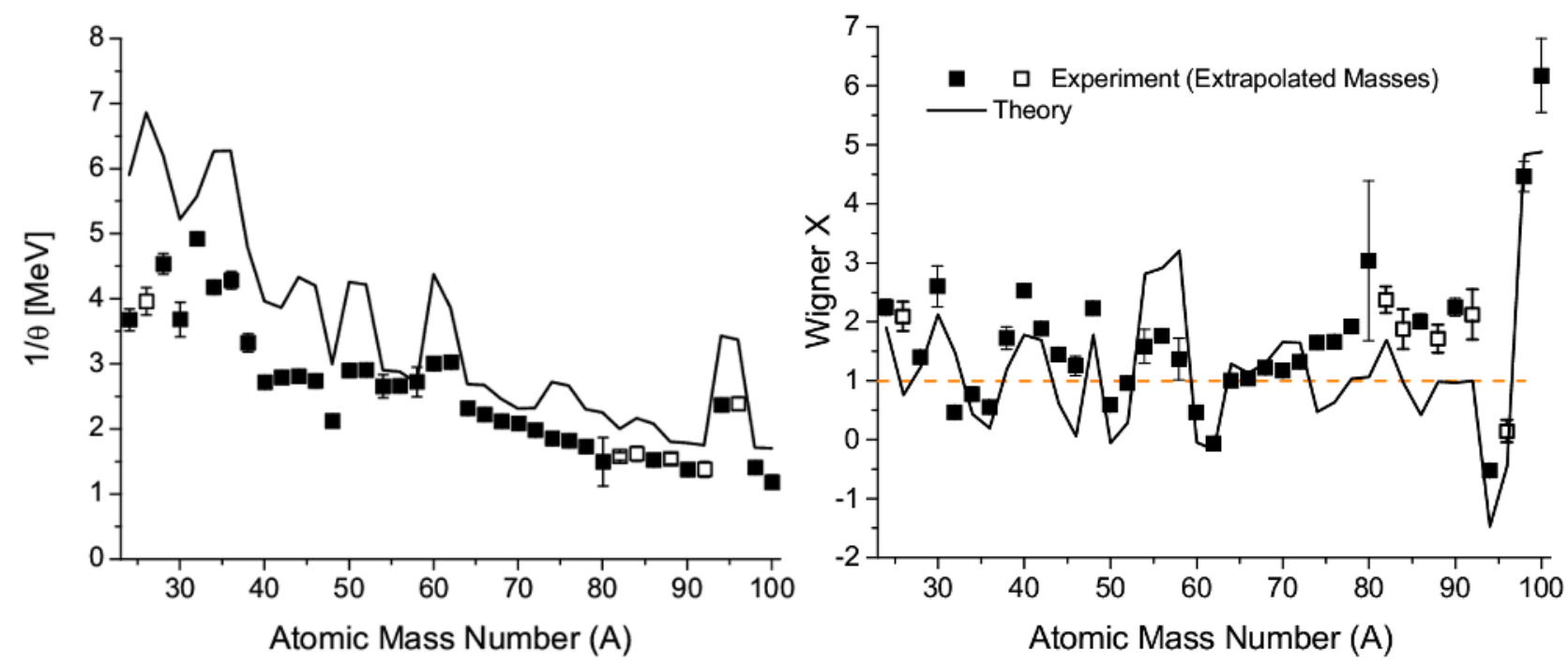

Figure 2. (Left) Slope of the isobar energies obtained from (8) using modified energies from [13] with the Coulomb energy removed. The solid line shows the calculations. (Right) The Wigner X obtained using modified energies from [13] with the Coulomb energy removed. The solid line shows the calculations. The orange dashed line indicates $X=1$

isospin invariance on the mean field level. The deformation in isospace gives rise to an isorotational band with energies $\propto T(T+1)$. The deformation is caused by the isovector pair field and the differences between the proton and neutron nuclear potentials to about equal parts. The limit $X=1$ corresponds to a regular isorotational band, which emerges if isospin is strongly broken by the pair field. Because the realistic pair field has only moderate strength, the bunching of the single particle levels, resulting from shell structure, causes strong fluctuations of the Wigner energy, which are fairly well described by the model. The remaining deviations can be attributed to inaccuracies of the calculated single particle energies.

A combination of an isorotational invariant effective interaction in the particle-hole channel with isovector pairing interaction is capable of reproducing the Wigner energy, provided the pairing correlations are treated beyond the mean field approximation and isospin is conserved. How to accomplish this for the present standard mean field approaches remains to be studied. In a future study we will address this question by comparing our results with approximations as e.g. isospin projected mean field solutions.

Supported by the DoE Grant DE-FG02-95ER4093.

\section{References}

[1] W. D. Myers and W. J. Swiatecki, Ann. Rev. Nucl. Part. Sci. 32, 309 (1982).

[2] E. Wigner, Phys. Rev. 51, 947 (1937).
[3] K. Neergard, Physical Review C 80, 044313 (2009).

[4] S. Goriely, N. Chamel, and J. M. Pearson, Phys. Rev. C 82, 035804 (2010).

[5] S. G. Frauendorf and J. A. Sheikh, Nuclear Physics A 645, 509 (1999).

[6] S. Frauendorf and J. A. Sheikh, Physica Scripta T88, 162 (2000).

[7] C. D. O’Leary et al., Phys. Rev. C 67, 021301 (2003).

[8] A. V. Afanasjev and S. Frauendorf, Phys. Rev. C 71, 064318 (2005).

[9] C. Andreoiu, C. E. Svensson, A. V. Afanasjev et al., Phys. Rev. C 75, 041301 (2007).

[10] J. Jänecke and T. O'Donnell, Physics Letters B 605, 87 (2005)

[11] W. Satula et al., Phys. Rev. Lett. 103, 012502 (2009).

[12] S. Nilsson et al., Shapes and Shells in Nuclear Structure (Cambridge University Press, 1995).

[13] G. Audi et al., Chinese Physics C 36, 1287 (2012).

[14] A. Bohr and B. Mottelson, Nuclear Structure I: Single-Particle Motion (World Scientific, 1999).

[15] S. Frauendorf, Nuclear Physics A 677, 115 (2000).

[16] I. Bentley and S. Frauendorf, Phys. Rev. C 83, 064322 (2011).

[17] A. O. Macchiavelli et al. Phys. Rev. C 61, 041303 (2000).

[18] J. Tuli, Evaluated nuclear structure data file", Data retrieved on: the 21st of December (2011).

[19] P. Vogel, Nuclear Physics A 662, 148 (2000). 